\title{
Information sharing and coordination in a vaccine supply chain
}

\author{
Yuqing $\operatorname{Pan}^{1} \cdot$ Chi To $\mathrm{Ng}^{1}$ (D) Ciwei Dong ${ }^{2}$ (D) T. C. E. Cheng ${ }^{1}$
}

Accepted: 18 January 2022

(c) The Author(s), under exclusive licence to Springer Science+Business Media, LLC, part of Springer Nature 2022

\begin{abstract}
Vaccination is a well-known method to protect the public against an epidemic outbreak, e.g., COVID-19. To this end, the government of a country or region would strive to achieve its target of vaccination coverage. Limited by the total vaccine capacity of public hospitals, the government may need to cooperate with private hospitals or clinics for more vaccination. Exploring in this paper government coordination of public and private resources for vaccination, we model a vaccine system consisting of a public hospital, a profit-maximizing private clinic, and self-interested individuals, under three scenarios: (1) without information sharing (concerning vaccine inventory and vaccine price), (2) with information sharing and subsidy, and (3) with information sharing and allocation. We find that, under scenario (1), the vaccine demand is fully satisfied by the public hospital and the private clinic cannot make any profit. Under scenario (2), the private clinic is willing to enter the vaccine market with a positive profit-maximizing vaccination coverage. Under scenario (3), the socially optimal vaccination coverage may be lower than that under scenario (1). Moreover, we conduct a sensitivity analysis to generate practical implications of the research findings for vaccination policy-making. Our results provide both theoretical and managerial insights on vaccine supply decision, government intervention, and vaccination coverage.
\end{abstract}

Keywords Vaccine supply chain · Information sharing · Subsidy scheme · Government coordination

\section{Introduction}

Vaccination is one of the most cost-effective medical interventions to prevent seasonal influenza infection (CDC 2019). Unfortunately, the vaccination coverage is always undesirably low in real practice. On the supply side, production uncertainty is considered as the main cause of the low coverage (Deo and Corbett 2009). On the demand side, the positive

\footnotetext{
Ciwei Dong

dongciwei@zuel.edu.cn

1 Logistics Research Centre, Department of Logistics and Maritime Studies, The Hong Kong Polytechnic University, Hung Hom, Kowloon, Hong Kong SAR, China
}

2 School of Business Administration, Zhongnan University of Economics and Law, Wuhan 430073, China 
externalities of vaccination, i.e., the indirect benefits accruing to other individuals, affect individuals' vaccination decisions (Galvani et al. 2007).

To safeguard the public against flu infection, the government is committed to increasing the overall flu vaccination coverage and enhancing the disease's prevention (Hong Kong Government News 2020a). The government is usually one of the key parties in the vaccine supply chain. A typical vaccine supply chain consists of a manufacturer, a government that makes vaccine orders, and self-interested individuals (Adida et al. 2013; Arifoğlu et al. 2012). Meanwhile, in a vaccine market (e.g., Hong Kong), there exist not only public hospitals, but also private clinics, which the government cannot make orders for. Our interview with a public health researcher reveals that, in the vaccine market of Hong Kong, the government and private clinics make vaccine orders to manufacturers before the flu season and the orders are always satisfied. But vaccine shortage still happens in private clinics (Cheng et al. 2020). To improve vaccination coverage, the government needs to cooperate with private clinics to eliminate supply shortage and stimulate vaccine demand.

For example, the Hong Kong government has implemented policies to improve vaccination coverage. The Centre for Health Protection of Hong Kong has introduced the Vaccination Programme for more than ten years. Under this programme, the priority group (e.g., all the citizens aged 65 or above) can take vaccination for free in public hospitals (Center of Health Protection 2020). However, limited by the total vaccine capacity of public hospitals, the vaccination coverage is not high enough to keep the whole population in a safe status (Chief Secretary for Administration of the HKSAR 2021). Therefore, it is necessary for the government to encourage residents to take vaccination in private clinics. A few years ago, the Hong Kong government launched the "Vaccination Subsidy Scheme", under which the government provides subsidy to private clinics for the vaccines they have administered to qualified citizens. As a result, the private clinics ordered more vaccines and the vaccine price decreased, which stimulated people to take vaccination (Hong Kong Government News 2020b). In addition, in 2020, people were afraid of the double infections of COVID-19 and influenza. The private clinics in Hong Kong faced an unprecedented flu vaccine shortage (Cheng et al. 2020). Some experts suggested that the government collaborated with private doctors and allocated vaccines to private clinics. According to the Medical Association, the Secretary for Food and Health approved an agreement with vaccine manufacturers to distribute part of the vaccine supply to private doctors (Hong Kong Government News 2020a). Given the vaccination subsidy scheme and government allocation scheme, it is unclear as to which scheme is better, in terms of private clinics' profitability and vaccination coverage.

To study the effectiveness of the two schemes, we first model a vaccine system without information sharing as the benchmark. In this vaccine system, there are a profit-maximizing private clinic, a social-cost-minimizing public hospital, and self-interested individuals. Each of the public hospital and private clinic decides its vaccine inventory and vaccine price independently with no knowledge of the other's inventory and price information. We show that restricted by limited information and insufficient public health care resources, some problems emerge from the vaccine system without information sharing as follows: (1) The vaccine demand is fully satisfied by the public hospital and the private clinic cannot make any profit. As such, the private clinic has no incentive to order vaccines, which is adverse to the vaccine market's development. (2) The public hospital allocates too many medical resources to the vaccination programme, which might undermine the other parts of the public health care system.

We model the "Vaccination Programme" and the "Vaccination Subsidy Scheme" as a vaccine system with information sharing and subsidy, which resembles the vaccine market in Hong Kong. The public hospital only provides free vaccines to the priority group and 
decides the subsidy for the private clinic for the vaccines they have administered to qualified citizens. Observing the vaccine inventory in the public hospital and vaccine subsidy, the private clinic decides its vaccine inventory and vaccine price. We find that, in the vaccine system with information sharing and subsidy, the private clinic is willing to order vaccines and enter the vaccine market. When the vaccine subsidy is less than or equal to the vaccine cost, the profit-maximizing vaccine inventory cannot satisfy half of the demand of the non-priority group. As the range of the priority group decreases, the profit-maximizing vaccination coverage decreases and the profit-maximizing price increases. This is because when the range of the priority group decreases, some customers with high infection disutility that cannot get the vaccine in the public hospital are willing to pay a high price for the vaccine in the private clinic. The private clinic increases its vaccine price to maximize its profit and does not serve the customers with low infection disutility any more. Moreover, as the vaccine subsidy increases, the profit-maximizing inventory increases and the profit-maximizing price decreases. This implies that vaccine subsidy can stimulate vaccine supply and demand simultaneously. Besides, as the range of the priority group decreases, the socially optimal subsidy decreases and the vaccine demand of the non-priority group increases.

Moreover, we model the vaccine system with information sharing and allocation to study the effectiveness of this cooperation scheme. Under this scheme, the public hospital provides free vaccines to the priority group and the private clinic. The private clinic makes profit from administering the vaccines to qualified citizens, but the vaccine inventory and vaccine price of the private clinic are decided by the public hospital. In this vaccine system, the public hospital can increase the vaccine inventory in the private clinic in order to increase the supply and decrease the vaccine price to induce more demand, so increasing the vaccination coverage. The vaccination coverage is not affected by the range of the priority group because all the vaccines are ordered by the public hospital. As the vaccine cost increases, the socially optimal vaccine inventory decreases and the vaccine price increases.

Furthermore, we conduct a sensitivity analysis to study the effects of the vaccine cost. In the vaccine system without information sharing, both the socially optimal coverage of the public hospital and the profit-maximizing coverage of the private clinic decrease with the vaccine cost. This is because as the vaccine cost increases, the social cost increases and the profit of the private clinic decreases. The socially optimal coverage is always higher than the profit-maximizing coverage because the public hospital considers not only the profit from selling vaccines, but also the infection cost of the non-vaccinated individuals. In both vaccine systems with information sharing, the socially optimal coverage decreases with the vaccine cost, where the decreasing rate in the vaccine system with allocation is higher than that in the vaccine system with subsidy. This is because in the vaccine system with allocation, all the vaccines are ordered by the public hospital, while in the vaccine system with subsidy, the public hospital only orders vaccines for itself. So the socially optimal coverage in the vaccine system with allocation is more affected by the vaccine cost. When the vaccine cost is low, the socially optimal coverage in the vaccine system with allocation is higher than that in the vaccine system with subsidy. But when the vaccine cost is high, the socially optimal coverage in the vaccine system with allocation is lower than that in the vaccine system with subsidy.

We organize the rest of the paper as follows: In Sect. 2 we review the related literature to position our study. In Sect. 3 we introduce the model and discuss the assumptions. In Sect. 4 we analyze the vaccine system without information sharing. In Sect. 5 we study the vaccine system with information sharing and subsidy. In Sect. 6 we model the vaccine system with information sharing and allocation. Finally, in Sect. 7, we conclude the paper and suggest topics for future research. We provide the proofs of all the results in the Appendix. 


\section{Literature review}

Vaccination is an important measure in public health policy through which the government seeks to achieve a high immunization level. However, due to supply uncertainties and insufficient incentives of taking vaccination, the vaccination coverage is often below the socially optimal level (Duijzer et al. 2018). Many studies consider government coordination in the vaccine market. To improve vaccination coverage, the manufacturer needs to produce adequate quantities of the vaccine (Deng et al. 2008). Chick et al. (2008) studied several types of contracts with the objective of maximizing the benefits of the government and the manufacturer simultaneously. Dai (2015) and Dai et al. (2016) indicated that the existing contracts do not consider the supply inefficiency resulting from late delivery. They proposed a new contract to coordinate vaccine supply with on-time delivery. Arifoğlu et al. (2012) studied the vaccine supply chain with rational consumer behavior. Self-interested individuals make vaccination decisions considering infection risks and vaccine prices. In view of the fact that giving subsidies to vaccinated individuals and taxing non-vaccinated individuals can induce vaccine demand, (Brito et al. 1991). Demirci and Erkip (2020) adopted the bilevel programming approach to study the intervention problem for a vaccine market. Extending coordination to affect both the supply and demand sides, Adida et al. (2013) proposed a two-side subsidy mechanism depending on the vaccination coverage to achieve the socially optimal coverage. Arifoğlu and Tang (2021) studied the vaccine supply chain as a sequential game. They developed a two-sided incentive programme to eliminate the inefficiencies on both the supply and demand sides. However, there are few studies considering the private retailer in the vaccine market. We exclude production uncertainty, which is not a serious problem in some vaccine markets (e.g., Hong Kong), and consider the not-for-profit public hospital and for-profit private clinic as the vaccine retailers.

Vaccines are examples of public interest goods, whose demands are influenced by the related externalities and prices. The positive externality effect, i.e., vaccination not only protects the vaccinated people, but also decreases the infection probability of the non-vaccinated people by decreasing their contacts with the infected people, impacts consumers' vaccination decisions (Brito et al. 1991). Consumers are utility maximizing and forward-looking (Aviv and Pazgal 2008; Su and Zhang 2008). Self-interested individuals will compare the vaccine price with the expected infection cost and make vaccination decisions (Mamani et al. 2012). Pan et al. (2021) studied the effect of the free-riding behaviour on vaccination coverage, considering customer regret. Xie et al. (2021) analyzed the government subsidy on the R\&D of vaccine products with a risk-averse buyer. Governments in developing countries often dictate the retail prices of subsidized food and drugs (Tuck and Lindert 1996), which is commonly assumed in the related studies on the vaccine market (see, e.g., Adida et al. 2013; and Arifoğlu et al. 2012). But in some vaccine markets (e.g., Hong Kong), the government cannot control the vaccine prices charged by private clinics. Therefore, similar to Erhun et al. (2008) and Cho and Tang (2013), we consider the case where the private clinic decides the vaccine price and faces a price-sensitive demand.

Research on public-private partnership has enabled a better understanding of the relationship between the public and private sectors. Public-private partnership refers to the cooperative relationship between the public and private sectors for efficient provision of public goods. Besley and Ghatak (2001) studied the ownership structure of public products between the public and private sectors, and proposed public-private cooperation. Kivleniece and Quelin (2012) determined the value creation based on a theoretical model of two conceptual public-private structural alternatives. Iossa and Martimort (2015) compared several 
existing incentives for public-private partnership and derived the optimal contract. They presented a basic model of procurement in a multi-task environment, in which a risk-averse firm makes non-contractible efforts on cost reduction and quality improvement. Berenguer et al. (2017) studied the effects of subsidy on for-profit and not-for-profit organizations in a vaccine market with uncertain demand. Lin et al. (2022) considered influenza vaccine supply chain coordination in a centralized system and a decentralized system. Besides subsidy, the government might also allocate the vaccine to private clinics to improve vaccine supply. Differing from the above papers, we study and compare both the subsidy and allocation mechanisms.

\section{Modelling}

We consider a population of $N$ individuals. Infected individuals incur an expected infection disutility $\delta$ (Meltzer et al. 1999; Galvani et al. 2007), with probability density function $g($.) and cumulative probability function $G($.). Similar to Arifoğlu and Tang (2021), we consider $\delta$ follows an uniform distribution in $[0, \bar{\delta}]$. We assume that the vaccine is perfectly effective, i.e., all the vaccinated individuals are immunized against the infection (Brito et al. 1991; Arifoğlu et al. 2012). The non-vaccinated individuals may be infected with probability $P(f)$, which is continuous and non-increasing in $f \in[0,1]$, the vaccinated fraction of the population. Similar to Brito et al. (1991), we assume that $P($.$) is common knowledge. In the literature,$ the expected number of infected people is usually derived as $P(f)=\max \left\{1-\frac{1}{R_{0}}-f, 0\right\}^{1}$ (Mamani et al. 2012). Referring to this estimation, we consider $\frac{d^{2} P(f)}{d f^{2}}=0$. Then the expected number of infected people in the population, i.e., $N(1-f) P(f)$, is a convex decreasing function of $f$. Individuals take vaccination in the public hospital or private clinic at different vaccine prices. We use $r_{h}$ and $r_{c}$ to represent the vaccine prices in the public hospital and private clinic, respectively. To exclude the case where no one is willing to pay for the vaccine in the public hospital or private clinic, we assume that the vaccine price in any case is less than $\bar{\delta} P(0)$. When people can take vaccination in either the public hospital or the private clinic, we assume that they will choose the vaccine that has a lower price. The public hospital operates for public health, which aligns with the government's objective, while the private clinic operates for profit. Thus, we consider in this paper that the objective of the public hospital is to minimize the social cost, whereas the objective of the private clinic is to maximize its profit.

Governments in some countries or areas (e.g., Hong Kong) have launched different programmes to improve the vaccination coverage. We model and compare such programmes to derive management insights for vaccine market coordination. To explore the effectiveness of different measures to promote vaccination, we model a non-cooperative vaccine market shown in Fig. 1 as the benchmark. The manufacturer charges the vaccine cost (per vaccine) $c$ to the public hospital and private clinic. The public hospital (private clinic) makes decisions independently without sharing any information about its vaccine inventory and vaccine price to the private clinic (public hospital). Specifically, the sequence of events in the vaccine system without information sharing is as follows:

- Stage 1: The public hospital decides its vaccine inventory $q_{h}$ and vaccine price $r_{h}$ to minimize the social cost, without any information on $q_{c}$ and $r_{c}$. Meanwhile, the private clinic decides its vaccine inventory $q_{c}$ and vaccine price $r_{c}$ to maximize its profit, without any information on $q_{h}$ and $r_{h}$.

$1 R_{0}$ represents the basic reproduction number and is a measure of the infectiousness of a disease (Anderson and May 1992; Murray 1993). 
Fig. 1 Non-cooperative vaccine market

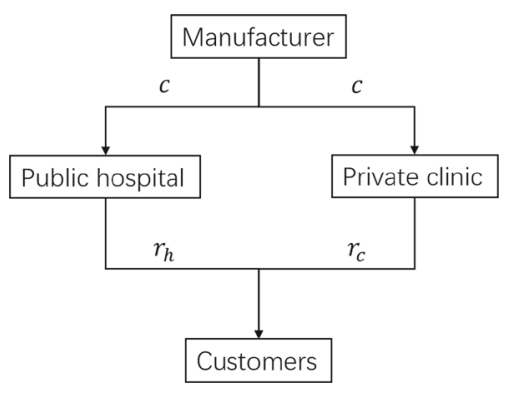

- Stage 2: Observing the vaccine prices in the public hospital and private clinic, individuals decide whether or not and where to take vaccination.

Restricted by limited information sharing and insufficient public health care resources, the vaccine system without information sharing is hard to achieve the socially optimal vaccination coverage. The government needs to implement some policies to improve the vaccination coverage. For example, the Centre for Health Protection of Hong Kong has launched the Vaccination Programme for more than ten years, under which public hospitals provide free vaccines to the priority group rather than the non-priority group (Center of Health Protection 2020). It provides social benefits to the priority group while keeping the public hospitals from being overloaded. The priority group is characterized with a higher mortality and morbidity risk than the non-priority group (Meltzer et al. 1999; Galvani et al. 2007). To ensure analytical tractability, we prioritize individuals based on their infection disutility (Arifoğlu et al. 2012), whereby the individuals with infection disutility $\delta \in[\beta, \bar{\delta}]$ are in the priority group. Otherwise, they are in the non-priority group. The public hospital orders $N[\bar{G}(\beta)]$ vaccines for the priority group. Individuals always prefer a vaccine that has a lower price. So all the individuals in the priority group, i.e., $\delta \in[\beta, \bar{\delta}]$, take free vaccination in the public hospital. The range of the priority group is restricted by the public hospital's capacity planning and the government's fiscal policy. Thus we do not discuss the decision on $\beta$ in this paper.

In addition to the policy for the priority group, the government proposes several cooperation schemes with the private clinic to improve the vaccination coverage for the non-priority group. The private clinic operates for profit and does not consider the social benefits. For example, in the last few years, the Hong Kong government has run the "Vaccination Subsidy Scheme" to stimulate vaccine supply and demand at the same time (Hong Kong Government News 2020b). Under the Vaccination Subsidy Scheme, individuals in the non-priority group can take vaccination in the private clinic at the vaccine price $r_{s}$ and the private clinic receives $r_{s}+s$ per vaccine sold, where $s$ is the subsidy per vaccine from the government. Figure 2 shows the vaccine system with information sharing and subsidy. The sequence of events is as follows:

- Stage 1: Given that the vaccine inventory in the public hospital is $N \bar{G}(\beta)$, the government decides the subsidy $s$ per vaccinated person for the private clinic.

- Stage 2: With the information on the vaccine inventory in the public hospital and the subsidy, i.e., $N \bar{G}(\beta)$ and $s$, the private clinic decides the vaccine inventory $q_{s}$ and vaccine price $r_{s}$.

- Stage 3: Given $r_{s}$, individuals make vaccination decisions. When the vaccine inventory in the private clinic is less than the vaccine demand, every individual in the non-priority group has the same probability of being vaccinated. 
Fig. 2 Vaccination subsidy scheme

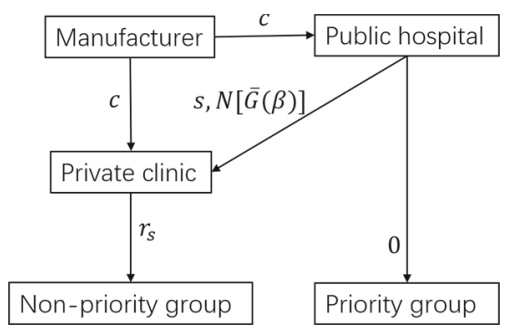

Fig. 3 Government allocation scheme

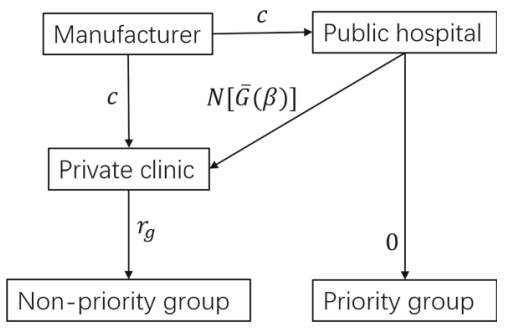

In the past two years, people were afraid of the double infections of COVID-19 and influenza. Private clinics in Hong Kong faced an unprecedented flu vaccination shortage (Cheng et al. 2020). Some experts suggested that the government worked with private doctors and allocated part of the vaccines to private clinics. According to the Medical Association in Hong Kong, the Secretary for Food and Health approved an agreement with vaccine manufacturers to distribute part of the vaccines to private clinics, but the clinics must comply with government regulations and must not increase the price of the vaccine (Hong Kong Government News 2020a). In this way, the non-priority group can take vaccination in cooperating private clinics at the price $r_{g}$ set by the government. This scheme has not been proposed officially. We study this vaccine system with information sharing and allocation as depicted in Fig. 3. The sequence of events is as follows:

- Stage 1: Given that the vaccine inventory in the public hospital is $N \bar{G}(\beta)$, the public hospital decides the vaccine inventory $q_{g}$ and vaccine price $r_{g}$ for the private clinic. The private clinic receives $r_{g}$ per vaccinated person.

- Stage 2: With the information of vaccine price $r_{g}$, individuals make vaccination decisions. When the vaccine inventory in the private clinic is less than the vaccine demand, every individual in the non-priority group has the same probability of getting vaccinated.

Table 1 summarizes the notation in this paper.

\section{Vaccine system without information sharing}

We model a vaccine system consisting of a public hospital that operates for public benefits, a profit-maximizing private clinic, and self-interest individuals that make their own vaccination decisions. There is no cooperation or information sharing between the public hospital and private clinic, and they make decisions without knowledge of the other's inventory and price information. First, each of the public hospital and private clinic independently decides its own vaccine inventory and vaccine price. Second, individuals make their own vaccination decisions. We use the subscript " $W$ " to denote the situation of the non-cooperative vaccine market. 
Table 1 Notation

\begin{tabular}{|c|c|}
\hline$\delta$ & An individual's infection disutility \\
\hline $\bar{\delta}$ & The highest infection disutility \\
\hline$g()$. & Probability density function of $\delta$ \\
\hline$G()$. & Cumulative probability function of $\delta$ \\
\hline$f$ & Vaccination coverage for the population \\
\hline$P(f)$ & Infection probability for the non-vaccinated group with vaccination coverage $f$ \\
\hline$N$ & Number of people in the population \\
\hline$c$ & $\begin{array}{l}\text { Vaccine cost (per vaccine) charged by the manufacturer to the public hospital and private } \\
\text { clinic. }\end{array}$ \\
\hline$r_{h}$ & Vaccine price of the public hospital in the non-cooperative vaccine market \\
\hline$q_{h}$ & Vaccine inventory in the public hospital in the non-cooperative vaccine market \\
\hline$r_{c}$ & Vaccine price of the private clinic in the non-cooperative vaccine market \\
\hline$q_{c}$ & Vaccine inventory in the private clinic in the non-cooperative vaccine market \\
\hline$\beta$ & The infection disutility of the marginal people between the priority and non-priority groups \\
\hline$r_{S}$ & Vaccine price of the private clinic under the vaccination subsidy scheme \\
\hline$s$ & The subsidy per vaccinated person from the government to the private clinic \\
\hline$q_{s}$ & Vaccine inventory in the private clinic under the vaccination subsidy scheme \\
\hline$r_{g}$ & Vaccine price in the private clinic under the government allocation scheme \\
\hline$q_{g}$ & Vaccine inventory in the private clinic under the government allocation scheme \\
\hline$\delta_{h}$ & The infection disutility of the marginal customer with respect to $r_{h}$ \\
\hline$\delta_{c}$ & The infection disutility of the marginal customer with respect to $r_{c}$ \\
\hline$\phi_{w}$ & The probability of being vaccinated in the vaccine system without information sharing \\
\hline$f_{w}$ & The vaccination coverage in the vaccine system without information sharing \\
\hline$S C_{w}$ & The social cost in the vaccine system without information sharing \\
\hline$\pi_{w}$ & The profit of the private clinic in the vaccine system without information sharing \\
\hline$\delta_{S}$ & The infection disutility of the marginal customer with respect to $r_{s}$ \\
\hline$\phi_{s}$ & $\begin{array}{l}\text { The probability of being vaccinated in the vaccine system with information sharing and } \\
\text { subsidy }\end{array}$ \\
\hline$f_{s}$ & The vaccination coverage in the vaccine system with information sharing and subsidy \\
\hline$S C_{S}$ & The social cost in the vaccine system with information sharing and subsidy \\
\hline$\pi_{S}$ & The profit of the private clinic in the vaccine system with information sharing and subsidy \\
\hline$\delta_{g}$ & The infection disutility of the marginal customer with respect to $r_{g}$ \\
\hline$\phi_{g}$ & $\begin{array}{l}\text { The probability of being vaccinated in the vaccine system with information sharing and } \\
\text { allocation }\end{array}$ \\
\hline$f_{g}$ & The vaccination coverage in the vaccine system with information sharing and allocation \\
\hline$S C_{g}$ & The social cost in the vaccine system with information sharing and allocation \\
\hline & The profit of the private clinic in the vaccine system with information sharing and allocation \\
\hline
\end{tabular}




\subsection{Individuals' problem}

In the second stage of the game, observing the vaccine prices of the private clinic and public hospital, each individual decides whether and where to take vaccination. An individual with infection disutility $\delta$ that decides not to take vaccination will be healthy with probability $1-P(f)$. He will be infected with probability $P(f)$ and will cause infection disutility $\delta$. His expected cost of not taking vaccination is $\delta P(f)$. So, an individual with infection disutility $\delta$ is willing to pay for the vaccine in the public hospital when

$$
\delta P(f) \geq r_{h} .
$$

An individual with infection disutility $\delta$ is willing to pay for the vaccine in the private clinic when

$$
\delta P(f) \geq r_{c} .
$$

Clearly, in equilibrium, if an individual with infection disutility $\hat{\delta}$ is not willing to pay for the vaccine in the public hospital (or in the private clinic), then none of the individuals with $\delta<\hat{\delta}$ is willing to pay for the vaccine in the public hospital (or in the private clinic). Therefore, the marginal customer that is indifferent to taking vaccination in the public hospital (or in the private clinic) satisfies the following condition

$$
\delta_{h} P\left(\bar{G}\left(\delta_{h}\right)\right)=r_{h}\left(\delta_{c} P\left(\bar{G}\left(\delta_{c}\right)\right)=r_{c}\right),
$$

where $\bar{G}()=.1-G($.$) .$

Given $P\left(\right.$.) and $G(),. r_{h}$ and $r_{c}$ can be decided by $\delta_{h}$ and $\delta_{c}$, respectively. To facilitate the presentation, we set $\delta_{h}$ and $\delta_{c}$ as decision variables of the public hospital and private clinic, respectively. Once $\delta_{h}$ and $\delta_{c}$ are settled, we can derive the vaccine prices.

Lemma 1 In equilibrium, there is a unique $\delta_{h}\left(\delta_{c}\right)$ at which all the individuals with $\delta>\delta_{h}$ $\left(\delta>\delta_{c}\right)$ are willing to pay for the vaccine in the public hospital (or in the private clinic) and all the individuals with $\delta<\delta_{h}\left(\delta<\delta_{c}\right)$ are not willing to pay for the vaccine in the public hospital (or in the private clinic).

Lemma 1 implies the existence and uniqueness of the marginal customer in the public hospital (or in the private clinic). The public hospital and private clinic make their decisions independently, so we cannot directly characterize the relationship between $\delta_{h}$ and $\delta_{c}$. For clarity, we use $i$ ( $j$ ) to denote the public hospital or the private clinic with the lower (higher) vaccine price. Then we have

$$
r_{i}<r_{j}
$$

and

$$
\delta_{i}<\delta_{j}
$$

For an individual with infection disutility $\delta$, his probability of being vaccinated is

$$
\phi_{w}(\delta)= \begin{cases}\min \left\{1, \frac{q_{i}}{N\left[\bar{G}\left(\delta_{i}\right)\right]}+\frac{q_{j}}{N\left[\bar{G}\left(\delta_{j}\right)\right]}\right\} & \delta \geq \delta_{j}, \\ \min \left\{1, \frac{q_{i}}{N\left[\bar{G}\left(\delta_{i}\right)\right]}\right\} & \delta_{i} \leq \delta<\delta_{j}, \\ 0 & \delta \leq \delta_{i} .\end{cases}
$$


For an individual with $\delta P(\bar{G}(\delta)) \geq r_{j}$, he first tries to take the vaccine at price $r_{i}$. If he does not get the vaccine at a lower price, he will try to take the vaccine at a higher price $r_{j}$. All the individuals that are willing to pay for the same type of vaccine have the same probability of being vaccinated. So the vaccination coverage is

$$
f_{w}=\bar{G}\left(\delta_{j}\right) \min \left\{1, \frac{q_{i}}{N\left[\bar{G}\left(\delta_{i}\right)\right]}+\frac{q_{j}}{N\left[\bar{G}\left(\delta_{j}\right)\right]}\right\}+\left(G\left(\delta_{j}\right)-G\left(\delta_{i}\right)\right) \min \left\{1, \frac{q_{i}}{N\left[\bar{G}\left(\delta_{i}\right)\right]}\right\},
$$

where the first term is the vaccination coverage from the customers that are willing to pay $r_{j}$ for the vaccine and the second term is the vaccination coverage from the customers that are only willing to pay $r_{i}$ for the vaccine.

\subsection{Public hospital's problem}

In the first stage of the non-cooperative market, the public hospital and private clinic make decisions before the flu season without information sharing. So both make decisions based on their own information and do not consider the inventory and price of the other. The public hospital decides the vaccine price, determined by $\delta_{h}$, and the inventory $q_{h}$ to minimize the social cost, which includes the profit of selling the vaccine and individuals' utility. So the public hospital's problem is as follows:

$$
\begin{aligned}
\min S C_{w}\left(q_{h}, \delta_{h}\right)= & c q_{h}-r_{h} \min \left\{N \bar{G}\left(\delta_{h}\right), q_{h}\right\}+\int_{0}^{\delta_{h}} \delta N P\left(f_{w}\right) d G(\delta) \\
& +\int_{\delta_{h}}^{\bar{\delta}} \delta\left(1-\phi_{w}\right) N P\left(f_{w}\right) d G(\delta),
\end{aligned}
$$

where the first term is the cost of the vaccine, the second term is the revenue of the sold vaccines, and the third term and the fourth term are individuals' infection disutility. The public hospital tries to minimize the social cost. Solving the optimization problem in Eq. (6), we obtain the following result that characterizes the socially optimal decision for the public hospital.

Proposition 1 The socially optimal inventory for the public hospital $q_{h}^{*}$ is $N \bar{G}\left(\delta_{h}^{*}\right)$ and the socially optimal price $r_{h}^{*}$ is $\delta_{h}^{*} P\left(\bar{G}\left(\delta_{h}^{*}\right)\right)$, where $\delta_{h}^{*} \geq \frac{\bar{\delta}}{3}$ and satisfies $g\left(\delta_{h}^{*}\right) P\left(\bar{G}\left(\delta_{h}^{*}\right)\right)\left(3 \delta_{h}^{*}-\bar{\delta}\right)+\frac{d P\left(\bar{G}\left(\delta_{h}^{*}\right)\right)}{d \delta_{h}^{*}}\left(\int_{0}^{\delta_{h}^{*}} \delta d G(\delta)-\delta_{h} g\left(\delta_{h}^{*}\right)\left(\bar{\delta}-\delta_{h}^{*}\right)\right)-c g\left(\delta_{h}^{*}\right)=0$.

Proposition 1 states the socially optimal inventory and price for the public hospital. It suggests that the socially optimal vaccine inventory would make at most two thirds of the population being vaccinated. The vaccine price affects the vaccine demand and makes it equal to the vaccine inventory. When the vaccine price is higher than $r_{h}^{*}$, as vaccine price increases, vaccine demand decreases and individuals' utility decreases. When vaccine price is lower than $r_{h}^{*}$, as the vaccine price decreases, the vaccine demand increases, the probability of being vaccinated for individuals with high infection disutility decreases, and the social cost increases. Besides, we obtain that $\delta_{h}^{*}$ increases in $c$. Clearly, as the vaccine cost increases, the socially optimal inventory decreases and the socially optimal vaccine price increases.

It is worth noting that, in real practice, the public hospitals in some cities provide free medical service to residents. So we consider the special case where the public hospital provides free vaccines and the corresponding problem is as follows: 


$$
\min S C_{w}^{\prime}\left(q_{h}^{\prime}, \delta_{h}^{\prime}\right)=c N\left(\bar{G}\left(\delta_{h}^{\prime}\right)\right)+\int_{0}^{\bar{\delta}} \delta(1-\phi) N P\left(f_{w}^{\prime}\right) d G(\delta) .
$$

The following result characterizes the property of the socially optimal decision for free vaccines.

Lemma 2 The socially optimal inventory for free vaccines is $N\left[\bar{G}\left(\delta_{h}^{\prime *}\right)\right]$, where $\delta_{h}^{\prime *}$ satisfies $c-\delta_{h}^{\prime *} P\left(\bar{G}\left(\delta_{h}^{*}\right)\right)<0$.

Lemma 2 shows that free vaccines should be allocated to individuals with high infection disutility. Besides, the lowest expected infection cost for the vaccinated people should be higher than the vaccine cost.

\subsection{Private clinic's problem}

In the first stage of the game, the private clinic decides the vaccine inventory $q_{c}$ and vaccine price $\delta_{c}$ to maximize its profit. The private clinic's problem is as follows:

$$
\max \pi_{w}\left(q_{c}, \delta_{c}\right)=\delta_{c} P\left(\bar{G}\left(\delta_{c}\right)\right) \min \left\{q_{c}, N\left[\bar{G}\left(\delta_{c}\right)\right]\right\}-c q_{c} .
$$

Solving the optimization problem (8), we characterize the profit-maximizing decision for the private clinic in the following result.

Proposition 2 The profit-maximizing inventory for the private clinic $q_{c}^{*}$ is $N\left[\bar{G}\left(\delta_{c}^{*}\right)\right]$ and the profit-maximizing price $r_{c}^{*}$ is $\delta_{c}^{*} P\left(\bar{G}\left(\delta_{c}^{*}\right)\right)$, where $\delta_{c}^{*} \geq \frac{\bar{\delta}}{2}$ and satisfies $N g\left(\delta_{c}\right)$ $\left(P\left(\bar{G}\left(\delta_{c}\right)\right)\left(\bar{\delta}-2 \delta_{c}\right)+\delta_{c}\left(\bar{\delta}-\delta_{c}\right) \frac{d P\left(\bar{G}\left(\delta_{c}\right)\right)}{d \delta_{c}}+c\right)=0$.

The profit-maximizing decision for the private clinic suggests that the vaccine inventory would make at most half of the population being vaccinated. The vaccine price affects the vaccine demand and makes it equal to the vaccine inventory. When the vaccine price is higher than $r_{c}^{*}$, as the vaccine price increases, the vaccine demand decreases and the profit decreases. When the vaccine price is lower than $r_{c}^{*}$, as the vaccine price decreases, the profit decreases. Besides, as the vaccine cost increases, the profit-maximizing inventory decreases and the profit-maximizing vaccine price increases. Comparing Proposition 1 with Proposition 2, we derive the following result.

Corollary 1 In the non-cooperative market, the socially optimal vaccine inventory for the public hospital is higher than the profit-maximizing vaccine inventory for the private clinic, i.e., $q_{h}^{*}>q_{c}^{*}$, and the socially optimal vaccine price is lower than the profit-maximizing vaccine price, i.e., $\delta_{h}^{*}<\delta_{c}^{*}$.

Corollary 1 provides the relationship between the socially optimal decision for the public hospital and the profit-maximizing decision for the private clinic. Clearly, the public hospital considers not only profit but also individuals' utility. So the socially optimal vaccination coverage is higher than the profit-maximizing coverage. It implies that in the vaccine market without the public hospital, the vaccination coverage would not be socially optimal. By Eq. (5), Propositions 1 and 2, and Corollary 1, we derive the vaccination coverage in the non-cooperative vaccine market as

$$
f_{w}=\bar{G}\left(\delta_{h}^{*}\right) .
$$

From the above analyses, we find several problems in the vaccine system without information sharing as follows: 
(1) In the vaccine system without information sharing, all the vaccine demand will be satisfied by the public hospital and the private clinic cannot make any profit. So the private clinic has no incentive to order vaccines, which is adverse to the vaccine market's development.

(2) The public hospital allocates too many medical resources to the vaccination programme, which might undermine the other parts of the public health care system.

To deal with these problems, we study several types of cooperation schemes in the vaccine market in the following.

\section{Vaccine system with information sharing and subsidy}

As discussed in Sect. 3, some countries or cities (e.g., Hong Kong) now implement the vaccination programme under the vaccination subsidy scheme. Because of their limited capacity, the public hospitals in Hong Kong only order a small quantity of vaccines and provide free vaccines to the high-risk group, i.e., the priority group. The quantity of free vaccines is limited by the public hospitals' capacity planning and the government's fiscal policy, which does not change every year. So we do not discuss the decision on the range of the priority group in this paper. Besides, the government provides subsidy to private clinics as an incentive for them to order vaccines and serve the vaccine market. We use the subscript "s" to denote the vaccine system with information sharing and subsidy. We model the vaccination subsidy scheme in the following steps: First, given the priority group $N[\bar{G}(\beta)]$, the public hospital decides the subsidy per vaccine $s$ for the private clinic. Second, the private clinic decides the vaccine inventory and vaccine price. Third, individuals make their own vaccination decisions. We use backward induction to characterize the equilibrium of this three-stage game.

\subsection{Individuals' problem}

In the third stage of the game, all the individuals in the priority group, i.e., $\delta \geq \beta$, take free vaccination in the public hospital. Observing the vaccine price, the non-priority individuals decide whether or not to take vaccination in the private clinic. By Lemma 1, we find that the infection disutility of the marginal customer $\delta_{s}$ under the vaccination subsidy scheme satisfies the following condition

$$
\delta_{s} P\left(\bar{G}\left(\delta_{s}\right)\right)=r_{s} .
$$

For an individual with infection disutility $\delta$, his probability of being vaccinated is

$$
\phi_{s}(\delta)= \begin{cases}1 & \beta \leq \delta, \\ \min \left\{1, \frac{q_{s}}{N\left[\bar{G}\left(\delta_{s}\right)-\bar{G}(\beta)\right]}\right\} & \delta_{s} \leq \delta<\beta .\end{cases}
$$

A non-priority individual with $\delta_{s} \leq \delta<\beta$ is willing to pay $r_{s}$ for the vaccine in the private clinic. So the vaccination coverage is

$$
f_{s}=\min \left\{\bar{G}(\beta)+\frac{q_{s}}{N}, \bar{G}\left(\delta_{s}\right)\right\},
$$

where the first term is the normalized total vaccine supply in the public hospital and private clinic, and the second term is the normalized total vaccine demand. 


\subsection{Private clinic's problem}

In the second stage under the vaccination subsidy scheme, observing the public hospital's vaccine subsidy, the private clinic decides its vaccine inventory $q_{s}$ and vaccine price, determined by $\delta_{s}$, to maximize its profit. The public hospital gives the private clinic $s$ per vaccine sold. So the private clinic gets $r_{s}+s$ per vaccine sold. It follows that the private clinic's problem is as follows:

$$
\max \pi_{s}\left(q_{s}, \delta_{s}\right)=\left(r_{s}+s\right) \min \left\{q_{s},\left(N\left[\bar{G}\left(\delta_{s}\right)-\bar{G}(\beta)\right]\right)^{+}\right\}-c q_{s},
$$

where the first term is the total revenue of the sold vaccines and the second term is the total vaccine cost.

Solving the optimization problem (12), we obtain the following result.

Lemma 3 The profit-maximizing decision for the private clinic satisfies the condition $\delta_{s}^{*}<\beta$.

Lemma 3 implies that, under the vaccination subsidy scheme, the private clinic is willing to order vaccines and enter the vaccine market. The marginal customer is in the non-priority group. The following result characterizes the profit-maximizing decision for the private clinic under the vaccination subsidy scheme.

Proposition 3 The profit-maximizing vaccine inventory $q_{s}^{*}$ is $N\left[\bar{G}\left(\delta_{s}^{*}\right)-\bar{G}(\beta)\right]$ and the profitmaximizing vaccine price $r_{s}^{*}$ is $\delta_{s}^{*} P\left(\bar{G}\left(\delta_{s}^{*}\right)\right)$. When $c-s \geq 0, \frac{\beta}{2} \leq \delta_{s}^{*} \leq \beta$ and satisfies $P\left(\bar{G}\left(\delta_{s}\right)\right)\left(\beta-2 \delta_{s}\right)+\delta_{s}\left(\beta-\delta_{s}\right) \frac{d P\left(\bar{G}\left(\delta_{s}\right)\right)}{d \delta_{s}}+c-s=0$; when $c-s<0$, $\delta_{s}^{*}$ might be smaller than $\frac{\beta}{2}$.

Proposition 3 illustrates that the profit-maximizing decision achieves the equilibrium between vaccine inventory and vaccine demand. In this case, the profit-maximizing vaccination coverage achieves $\bar{G}\left(\delta_{s}^{*}\right)$. When the vaccine subsidy is less than or equal to the vaccine cost, i.e., $c-s \geq 0$, the profit-maximizing vaccine inventory cannot satisfy half of the demand of the non-priority group. As $\beta$ increases, $\delta_{s}^{*}$ increases. This implies that as the range of the priority group decreases, the profit-maximizing vaccination coverage decreases and the profit-maximizing price increases. This is because when the range of the priority group decreases, some customers with high infection disutility that cannot get the vaccine in the public hospital are willing to pay a high price for the vaccine in the private clinic. The private clinic increases its vaccine price to maximize its profit and does not serve customers with low infection disutility any more. When the vaccine subsidy is larger than the vaccine cost, i.e., $c-s<0$, the profit-maximizing vaccine inventory can satisfy more than half of the demand of the non-priority group. As the vaccine subsidy increases, the profitmaximizing inventory increases and the profit-maximizing price decreases. This shows that vaccine subsidy can stimulate vaccine supply and demand simultaneously.

Corollary 2 Comparing Propositions 2 and 3, we have

$$
\delta_{s}^{*} \leq \delta_{c}^{*}
$$

Clearly, under the vaccination subsidy scheme, the infection disutility of the marginal customer in the private clinic is lower than that under the vaccine system without information sharing, and the vaccine price in the private clinic will be lower than or equal to that in the vaccine system without information sharing, i.e., $r_{s}^{*} \leq r_{c}^{*}$. 


\subsection{Public hospital's problem}

By Proposition 3, we see that the vaccine subsidy affects the profit-maximizing decision. The public hospital decides the vaccine subsidy $s$ to minimize the social cost. So the public hospital's problem is

$$
\min S C_{s}(s)=s V_{c}+\int_{0}^{\delta_{s}} \delta N P(f) d G(\delta)+\int_{\delta_{s}}^{\beta} \delta\left(1-\phi_{s}\right) N P(f) d G(\delta),
$$

where the first term is the total subsidy for the private clinic, and the second and third terms are individuals' infection costs.

Given $q_{s}^{*}$ in Proposition 3, the objective function is changed to

$$
\min S C_{s}(s)=s q_{s}^{*}+\int_{0}^{\delta_{s}^{*}} \delta N P(f) d G(\delta) .
$$

The following result characterizes the socially optimal decision.

Proposition 4 The socially optimal subsidy for the government ${ }^{*}$ satisfies the condition

$$
N\left[\bar{G}\left(\delta_{s}^{*}\right)-\bar{G}(\beta)\right]+N \frac{d \delta_{s}^{*}}{d s}\left(\frac{d P(f)}{d \delta_{s}^{*}} \int_{0}^{\delta_{s}^{*}} \delta d G(\delta)+P(f) \delta_{s}^{*} g\left(\delta_{s}^{*}\right)\right)=0 .
$$

Proposition 4 shows the socially optimal subsidy. As $\beta$ increases, $s^{*}$ decreases. This indicates that as the range of the priority group decreases, the vaccine demand in the nonpriority group increases, so the socially optimal subsidy decreases. In this case, the public hospital provides the subsidy $s\left(N\left[\bar{G}\left(\delta_{S}\right)-\bar{G}(\beta)\right]\right)$ and charges the vaccine cost $c(N[\bar{G}(\beta)])$ to achieve the vaccination coverage $\bar{G}\left(\delta_{s}\right)$.

Comparing Proposition 1 with Proposition 4, we derive the following result.

Corollary 3 If $N\left[\bar{G}(\beta)-\bar{G}\left(\delta_{s}^{*}\right)\right] \frac{d s}{d \delta_{s}^{*}}-N g\left(\delta_{s}^{*}\right)\left(P\left(\bar{G}\left(\delta_{s}^{*}\right)\right)\left(\bar{\delta}-2 \delta_{s}^{*}\right)+\delta_{s}^{*}\left(\bar{\delta}-\delta_{s}^{*}\right) \frac{d P\left(\bar{G}\left(\delta_{s}^{*}\right)\right)}{d \delta_{s}^{*}}\right.$ $+c) \leq 0, \delta_{h}^{*} \geq \delta_{s}^{*}$; otherwise, $\delta_{h}^{*}<\delta_{s}^{*}$.

Corollary 3 compares the socially optimal vaccine price in the non-cooperative vaccine market with that in the vaccine system with information sharing and subsidy. The result is affected by the range of the priority group and the vaccine cost.

\section{Vaccine system with information sharing and allocation}

In this section we consider the case where the public hospital orders vaccines for the high-risk group, i.e., the priority group. Besides, the public hospital also orders and allocates vaccines to the private clinic. The private clinic does not order any vaccine, but it can make profit by providing vaccinations to individuals at the vaccine price decided by the public hospital. We use the subscript " $g$ " to denote the vaccine system with information sharing and allocation. In the first stage, the public hospital decides the vaccine inventory and vaccine price for the private clinic. In the second stage, individuals make vaccination decisions. 


\subsection{Individuals' problem}

In the second stage, observing the vaccine price, non-priority individuals decide whether or not to take vaccination in the private clinic. Similar to Eq. (9), the infection disutility of the marginal customer under the government allocation scheme satisfies the following condition

$$
\delta_{g} P\left(\bar{G}\left(\delta_{g}\right)\right)=r_{g} .
$$

Individuals in the priority group, i.e., $\delta \geq \beta$, take vaccination for free in the public hospital. Non-priority individuals with $\delta_{g} \leq \delta<\beta$ are willing to pay $r_{g}$ for vaccination in the private clinic. For an individual with infection disutility $\delta$, his probability of being vaccinated is

$$
\phi_{g}(\delta)= \begin{cases}1 & \beta \leq \delta, \\ \min \left\{1, \frac{q_{g}}{N\left[\bar{G}\left(\delta_{g}\right)-\bar{G}(\beta)\right]}\right\} & \delta_{g} \leq \delta<\beta .\end{cases}
$$

The vaccination coverage is

$$
f_{g}=\min \left\{\bar{G}(\beta)+\frac{q_{g}}{N}, \bar{G}\left(\delta_{g}\right)\right\} .
$$

The profit of the private clinic is

$$
\pi_{g}=r_{g} \min \left\{q_{g},\left(N\left[\bar{G}\left(\delta_{g}\right)-\bar{G}(\beta)\right]\right)^{+}\right\} .
$$

\subsection{Public hospital's problem}

In the first stage of the game, the public hospital decides $q_{g}$ and $\delta_{g}$ for the private clinic to minimize the social cost. So the public hospital's problem is

$$
\begin{aligned}
\min S C_{g}\left(q_{g}, \delta_{g}\right)= & c\left(N[\bar{G}(\beta)]+q_{g}\right)+\int_{0}^{\delta_{g}} \delta N P\left(f_{g}\right) d G(\delta) \\
& +\int_{\delta_{g}}^{\beta} \delta\left(1-\phi_{g}\right) N P\left(f_{g}\right) d G(\delta),
\end{aligned}
$$

where the first term is the total vaccine cost in the public hospital and private clinic, and the second and third terms are individuals' infection costs.

Solving the optimization problem (21), we characterize the socially optimal decision for the vaccine system with information sharing and allocation in the following result.

Proposition 5 The socially optimal inventory is $N\left[\bar{G}\left(\delta_{g}^{*}\right)-\bar{G}(\beta)\right]$ and the socially optimal price is $P\left(\bar{G}\left(\delta_{g}^{*}\right)\right) \delta_{g}^{*}$, where $\delta_{g}^{*}$ satisfies $c+\left(\frac{d P\left(\bar{G}\left(\delta_{g}^{*}\right)\right)}{d \bar{G}\left(\delta_{g}^{*}\right)} \int_{0}^{\delta_{g}^{*}} \delta d G(\delta)-P\left(\bar{G}\left(\delta_{g}^{*}\right)\right) \delta_{g}^{*}\right)=0$.

Proposition 5 implies that the vaccination coverage under the government allocation scheme is $\bar{G}\left(\delta_{g}\right)$. The vaccination coverage is not affected by $\beta$. This is because all the vaccines are ordered by the public hospital. As $c$ increases, $\delta_{g}$ increases. This indicates that as the vaccine cost increases, the socially optimal vaccine inventory decreases and the vaccine price increases. In total, the public hospital spends $c N\left[\bar{G}\left(\delta_{g}\right)\right]$ and achieves vaccination coverage $\bar{G}\left(\delta_{g}\right)$. Comparing Proposition 1 with Proposition 5, we derive the following result.

Corollary 4 If $P\left(\bar{G}\left(\delta_{g}^{*}\right)\right)\left(\bar{\delta}-2 \delta_{g}^{*}\right)+\delta_{g}^{*}\left(\bar{\delta}-\delta_{g}^{*}\right) \frac{d P\left(\bar{G}\left(\delta_{g}^{*}\right)\right)}{d \delta_{g}^{*}} \leq 0, \delta_{g}^{*} \leq \delta_{h}^{*}$; otherwise, $\delta_{g}^{*}>\delta_{h}^{*}$. 


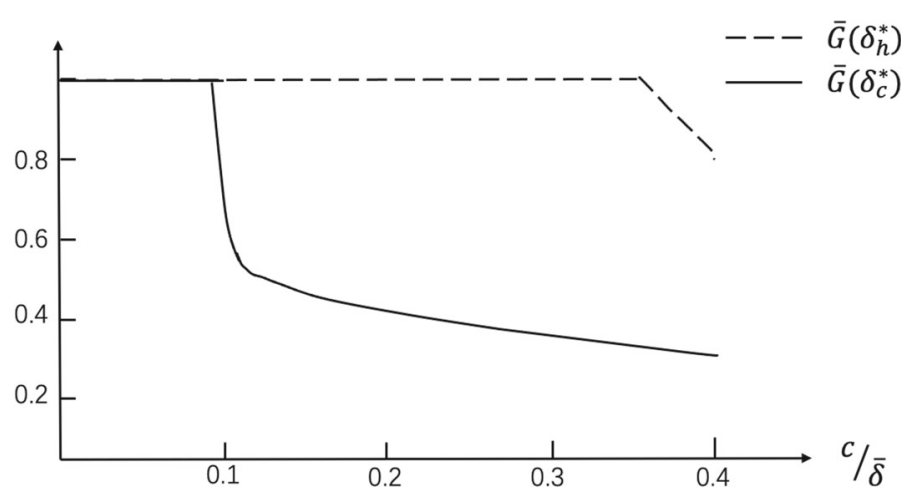

Fig. 4 Sensitivity analysis of the vaccine system without information sharing

Under the vaccination subsidy scheme, the public hospital provides the subsidy $s\left(N\left[\bar{G}\left(\delta_{S}\right)-\bar{G}(\beta)\right]\right)$ and charges the vaccine cost $c(N[\bar{G}(\beta)])$ to achieve the vaccination coverage $\bar{G}\left(\delta_{S}\right)$. Under the government allocation scheme, the public hospital spends $c N\left[\bar{G}\left(\delta_{g}\right)\right]$ and achieves the vaccination coverage $\bar{G}\left(\delta_{g}\right)$. Comparing $\delta_{s}^{*}$ in Proposition 4 with $\delta_{g}^{*}$ in Proposition 5, we derive the following result.

Corollary 5 If $\frac{d s}{d \delta_{s}^{*}}\left(\bar{G}(\beta)-\bar{G}\left(\delta_{s}^{*}\right)\right) \leq c N g(\delta), \delta_{s}^{*} \leq \delta_{g}^{*}$; otherwise, $\delta_{s}^{*}>\delta_{g}^{*}$, where $\frac{d s}{d \delta_{s}}=$ $-2 P\left(\bar{G}\left(\delta_{s}\right)\right)+2\left(\beta-2 \delta_{s}\right) \frac{d P\left(\bar{G}\left(\delta_{s}\right)\right)}{d \delta_{s}}+\delta_{s}\left(\beta-\delta_{s}\right) \frac{d^{2} P\left(\bar{G}\left(\delta_{s}\right)\right)}{d\left(\delta_{s}\right)^{2}}$.

Corollary 5 compares the socially optimal vaccine price in the vaccine system with information sharing and subsidy with that in the vaccine system with information sharing and allocation. The result is affected by the range of the priority group and the vaccine cost. Given that $\frac{d s}{d \delta_{s}} \leq 0$, as the vaccine subsidy increases, the vaccination coverage increases.

\subsection{Sensitivity analysis}

In this subsection we test the effects of the vaccine cost on the socially optimal coverage and the profit-maximizing coverage in different vaccine systems. Following Mamani et al. (2012) and Adida et al. (2013), we assume that $g($.$) follows a uniform distribution and$ $P(f)=1-f-\frac{1}{R_{0}}$, where $R_{0}=2$ (Wikipedia 2018). Following Arifoğlu et al. (2012), we assume $\bar{\delta}=100$. We set $\beta=0.6 \bar{\delta}$ and vary $c$. The result of the vaccine system without information sharing is shown in Fig. 4 and the result of the vaccine systems with information sharing is shown in Fig. 5.

From Fig. 4, we see that both the socially optimal coverage of the public hospital and the profit-maximizing coverage of the private clinic decrease with the vaccine cost. As the vaccine cost increases, the social cost increases and the profit of the private clinic decreases. The socially optimal coverage is always higher than the profit-maximizing coverage. The public hospital considers not only the profit from selling vaccines, but also the infection cost of the non-vaccinated individuals.

Figure 5 shows the effects of the vaccine cost on the vaccine system with information sharing and subsidy, and on the vaccine system with information sharing and allocation. In both vaccine systems, the socially optimal coverage decreases with the vaccine cost, where the decreasing rate in the vaccine system with allocation is higher than that in the vaccine system with subsidy. This is because in the vaccine system with allocation all the vaccines are 


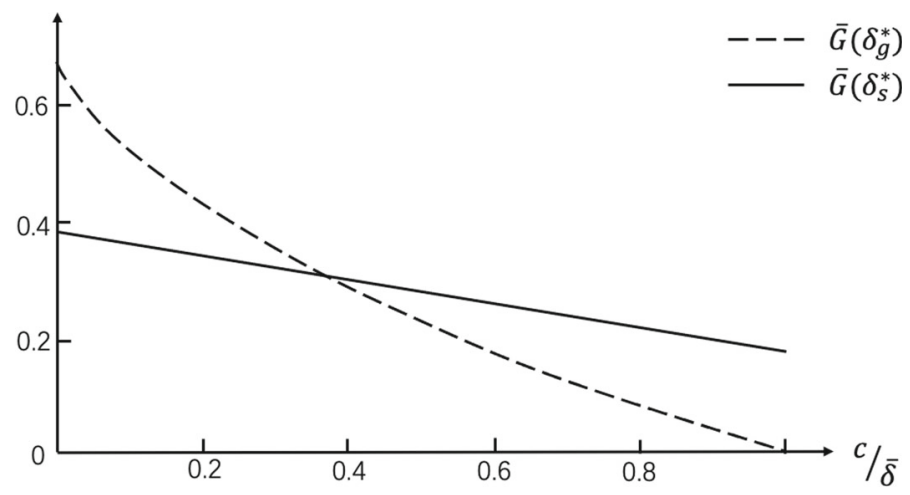

Fig. 5 Sensitivity analysis of the vaccine systems with information sharing

ordered by the public hospital, whereas in the vaccine system with subsidy the public hospital only orders vaccines for itself. So the socially optimal coverage in the vaccine system with allocation is affected more by the vaccine cost. When the vaccine cost is low, the socially optimal coverage in the vaccine system with allocation is higher than that in the vaccine system with subsidy. But when the vaccine cost is high, the socially optimal coverage in the vaccine system with allocation is lower than that in the vaccine system with subsidy.

\section{Conclusions}

In this paper we build models of a vaccine system to study the cooperation between the government and a private clinic for vaccination. In the vaccine system, there are a profit-maximizing private clinic, a public hospital that seeks to minimize the social cost, and self-interested individuals. We construct three models including a vaccine system without information sharing, a vaccine system with information sharing and subsidy, and a vaccine system with information sharing and allocation.

In the vaccine system without information sharing, the public hospital and private clinic decide the vaccine inventories and vaccine prices independently. Restricted by limited information sharing and insufficient public health care resources, some problems emerge in the vaccine system without information sharing. First, all the vaccine demand will be satisfied by the public hospital and the private clinic cannot make any profit. So the private clinic has no incentive to order vaccines, which is adverse to the vaccine market's development. Second, the public hospital allocates too many medical resources to vaccination, which might undermine the other parts of the public health care system.

In the vaccine system with information sharing and subsidy, we consider the "Vaccination Programme" and the "Vaccination Subsidy Scheme" based on real practice. The public hospital only provides free vaccines to the priority group. The public hospital decides the subsidy for the sold vaccines for the private clinic. Observing the vaccine inventory in the public hospital and vaccine subsidy, the private clinic decides its own vaccine inventory and vaccine price. 
We find that, in the vaccine system with information sharing and subsidy, the private clinic is willing to order vaccines and enter the vaccine market. When the vaccine subsidy is less than or equal to the vaccine cost, the profit-maximizing vaccine inventory cannot satisfy half of the demand of the non-priority group. As the range of the priority group decreases, the profit-maximizing vaccination coverage decreases and the profit-maximizing price increases. This is because when the range of the priority group decreases, some customers with high infection disutility that cannot get the vaccine in the public hospital are willing to pay a high price for the vaccine in the private clinic. The private clinic increases its vaccine price to maximize its profit and does not serve customers with low infection disutility any more. As the vaccine subsidy increases, the profit-maximizing inventory increases and the profitmaximizing price decreases. This implies that vaccine subsidy can stimulate vaccine supply and demand simultaneously. Besides, as the range of the priority group decreases, the vaccine demand of the non-priority group increases and the socially optimal subsidy decreases.

Following real practices, we further model the vaccine system with information sharing and allocation. The public hospital provides free vaccines to the priority group and private clinic. The private clinic makes profit from selling vaccines, where the vaccine inventory and vaccine price of the private clinic are decided by the public hospital. In this vaccine system, the public hospital can increase the vaccine inventory of the private clinic to increase the supply and decrease the vaccine price to increase the demand, thereby increasing the vaccination coverage. The vaccination coverage is not affected by the range of the priority group because all the vaccines are ordered by the public hospital. As the vaccine cost increases, the socially optimal vaccine inventory decreases and the vaccine price increases.

We also conduct a sensitivity analysis of the effects of the vaccine cost. In the vaccine system without information sharing, both the socially optimal coverage of the public hospital and the profit-maximizing coverage of the private clinic decrease with the vaccine cost. As the vaccine cost increases, the social cost increases and the profit of the private clinic decreases. The socially optimal coverage is always higher than the profit-maximizing coverage because the public hospital considers not only the profit from selling vaccines, but also the infection cost of the non-vaccinated individuals. In both vaccine systems with information sharing, the socially optimal coverage decreases with vaccine cost, where the decreasing rate in the vaccine system with allocation is higher than that in the vaccine system with subsidy. This is because in the vaccine system with allocation, all the vaccines are ordered by the public hospital, while in the vaccine system with subsidy, the public hospital only orders vaccines for itself. So the socially optimal coverage in the vaccine system with allocation is more affected by the vaccine cost. When the vaccine cost is low, the socially optimal coverage in the vaccine system with allocation is higher than that in the vaccine system with subsidy. But when the vaccine cost is high, the socially optimal coverage in the vaccine system with allocation is lower than that in the vaccine system with subsidy.

In this paper we assume that the public hospital and private clinic provide the same product and service. For future research, it would be interesting to consider the differences in service quality between the public hospital and private clinic. Besides, future research should also consider the cost of searching for vaccines in the vaccine system.

Acknowledgements The authors thank the editors and anonymous reviewers for their constructive comments on this study. The paper is partially supported by the Research Grants Council of Hong Kong under grant number PolyU 155033/19B, and the National Natural Science Foundation of China under grant number 71971215. 


\section{Appendix}

\section{Proof of Lemma 1}

$\frac{d \delta P(\bar{G}(\delta))}{d \delta}=P(\bar{G}(\delta))-\delta g(\delta) \frac{d P(\bar{G}(\delta))}{d \bar{G}(\delta)}>0$. When $\delta=0, \delta P(\bar{G}(\delta))=0$; when $\delta=\bar{\delta}$, $\delta P(\bar{G}(\delta))=\bar{\delta} P(0)>\max \left\{r_{h}, r_{c}\right\}$. So there exists a unique $\delta_{h}\left(\delta_{c}\right)$ satisfying $\delta_{h} P\left(\bar{G}\left(\delta_{h}\right)\right)=$ $r_{h}\left(\delta_{c} P\left(\bar{G}\left(\delta_{c}\right)\right)=r_{c}\right)$.

\section{Proof of Proposition 1}

Both the public hospital and private clinic make decisions based on their own information and do not consider the inventory and price of the other. In this case, they consider the other party's inventory as zero. By $\delta$ following an uniform distribution, we have $\frac{d g(\delta)}{d \delta}=0$. Then we have

$$
\begin{aligned}
& \frac{d P(\bar{G}(\delta))}{d \delta}=-g(\delta) \frac{d P(\bar{G}(\delta))}{d \bar{G}(\delta)} \geq 0 . \\
& \frac{d^{2} P(\bar{G}(\delta))}{d \delta^{2}}=-\frac{d g(\delta)}{d \delta} \frac{d P(\bar{G}(\delta))}{d \bar{G}(\delta)}-g(\delta) \frac{d^{2} P(\bar{G}(\delta))}{d(\bar{G}(\delta))^{2}} \frac{d \bar{G}(\delta)}{d \delta}=0 . \\
& \frac{d r_{h}}{d \delta_{h}}=\frac{d\left(\delta_{h} P\left(\bar{G}\left(\delta_{h}\right)\right)\right)}{d \delta_{h}}=P\left(\bar{G}\left(\delta_{h}\right)\right)-\delta_{h} g\left(\delta_{h}\right) \frac{d P\left(\bar{G}\left(\delta_{h}\right)\right)}{d \bar{G}\left(\delta_{h}\right)} \geq 0 . \\
& \frac{d \int_{0}^{\delta_{h}} \delta d G(\delta)}{d \delta_{h}}=G\left(\delta_{h}\right)+\delta_{h} g\left(\delta_{h}\right)-G\left(\delta_{h}\right)=\delta_{h} g\left(\delta_{h}\right) \geq 0 . \frac{d\left(P\left(\bar{G}\left(\delta_{h}\right)\right) \int_{0}^{\delta_{h}} \delta d G(\delta)\right)}{d \delta_{h}} \geq 0 . \\
& \left.\frac{d\left(\frac{q_{h}}{N\left[\bar{G}\left(\delta_{h}\right)-\bar{G}(\beta)\right]}\right.}{d \delta_{h}}\right) \\
& =\frac{q h}{N g(\delta)\left(\beta-\delta_{h}\right)^{2}} \geq 0 . \frac{d^{2}\left(\frac{q h}{N\left[\bar{G}\left(\delta_{h}\right)-\bar{G}(\beta)\right]}\right)}{d\left(\delta_{h}\right)^{2}}=\frac{2 q_{h}}{N g(\delta)\left(\beta-\delta_{h}\right)^{3}} \geq 0 .
\end{aligned}
$$

For $q_{h} \leq N \bar{G}\left(\delta_{h}\right), \frac{\partial S C}{\partial q_{h}}=c-r_{h}+\int_{0}^{\delta_{h}} \delta d G(\delta) \frac{d P(f)}{d f}+\int_{\delta_{h}}^{\bar{\delta}} \delta d G(\delta)\left(\left(1-\frac{q_{h}}{N \bar{G}\left(\delta_{h}\right)}\right) \frac{d P(f)}{d f}-\right.$ $\left.\frac{P(f)}{\bar{G}\left(\delta_{h}\right)}\right) \leq 0$; for $q_{h}>N \bar{G}\left(\delta_{h}\right), \frac{\partial S C}{\partial q_{h}}=c>0$. So $q_{h}^{*}=N \bar{G}\left(\delta_{h}\right)$. Given $q_{h}^{*}$, the function changes to

$$
\begin{aligned}
& S C\left(\delta_{h} \mid q_{h}^{*}\right)=\left(c-\delta_{h} P\left(\bar{G}\left(\delta_{h}\right)\right)\right) N \bar{G}\left(\delta_{h}\right)+\int_{0}^{\delta_{h}} \delta N P\left(\bar{G}\left(\delta_{h}\right)\right) d G(\delta) . \\
& \frac{\partial S C}{\partial \delta_{h}}=-N g\left(\delta_{h}\right)\left(P\left(\bar{G}\left(\delta_{h}\right)\right)\left(\bar{\delta}-2 \delta_{h}\right)+\delta_{h}\left(\bar{\delta}-\delta_{h}\right) \frac{d P\left(\bar{G}\left(\delta_{h}\right)\right)}{d \delta_{h}}+c\right) \\
& +N\left(\frac{d P\left(\bar{G}\left(\delta_{h}\right)\right)}{d \delta_{h}} \int_{0}^{\delta_{h}} \delta d G(\delta)+P\left(\bar{G}\left(\delta_{h}\right)\right) \delta_{h} g\left(\delta_{h}\right)\right) \\
& =N g\left(\delta_{h}\right) P\left(\bar{G}\left(\delta_{h}\right)\right)\left(3 \delta_{h}-\bar{\delta}\right)+N \frac{d P\left(\bar{G}\left(\delta_{h}\right)\right)}{d \delta_{h}}\left(\int_{0}^{\delta_{h}} \delta d G(\delta)-\delta_{h} g\left(\delta_{h}\right)\left(\bar{\delta}-\delta_{h}\right)\right)-c N g\left(\delta_{h}\right) . \\
& \int_{0}^{\delta_{h}} \delta d G(\delta)=\int_{0}^{\delta_{h}} \delta g(\delta) d \delta=g(.) \int_{0}^{\delta_{h}} \delta d \delta=\frac{1}{2} \delta_{h}^{2} g\left(\delta_{h}\right) \cdot \int_{0}^{\delta_{h}} \delta d G(\delta)-\delta_{h} g\left(\delta_{h}\right)\left(\bar{\delta}-\delta_{h}\right)=
\end{aligned}
$$
$\delta_{h} g().\left(\frac{3 \delta_{h}}{2}-\bar{\delta}\right)$.

When $3 \delta_{h}-\bar{\delta}<0$, we have $N g\left(\delta_{h}\right) P\left(\bar{G}\left(\delta_{h}\right)\right)\left(3 \delta_{h}-\bar{\delta}\right)<0$ and $N \frac{d P\left(\bar{G}\left(\delta_{h}\right)\right)}{d \delta_{h}}\left(\int_{0}^{\delta_{h}} \delta d G(\delta)\right.$ $\left.-\delta_{h} g\left(\delta_{h}\right)\left(\bar{\delta}-\delta_{h}\right)\right)<0$. Then $\frac{\partial S C}{\partial \delta_{h}}<0$, so $\delta_{h}^{*} \geq \frac{\bar{\delta}}{3}$.

$$
\begin{aligned}
& \frac{\partial^{2} S C}{\partial \delta_{h}^{2}}=-N g\left(\delta_{c}\right)\left(-2 P\left(\bar{G}\left(\delta_{c}\right)\right)+2\left(\bar{\delta}-2 \delta_{c}\right) \frac{d P\left(\bar{G}\left(\delta_{c}\right)\right)}{d \delta_{c}}+\delta_{c}\left(\bar{\delta}-\delta_{c}\right) \frac{d^{2} P\left(\bar{G}\left(\delta_{c}\right)\right)}{d\left(\delta_{c}\right)^{2}}\right) \\
& +N\left(\frac{d^{2} P\left(\bar{G}\left(\delta_{h}\right)\right)}{d \delta_{h}^{2}} \int_{0}^{\delta_{h}} \delta d G(\delta)+\frac{d P\left(\bar{G}\left(\delta_{h}\right)\right)}{d \delta_{h}} \delta_{h} g\left(\delta_{h}\right)+\frac{d P\left(\bar{G}\left(\delta_{h}\right)\right)}{d \delta_{h}} \delta_{h} g\left(\delta_{h}\right)+P\left(\bar{G}\left(\delta_{h}\right)\right) g\left(\delta_{h}\right)\right) \\
& =2 N g\left(\delta_{c}\right) P\left(\bar{G}\left(\delta_{c}\right)\right)+2 N g(\delta) \frac{d P\left(\bar{G}\left(\delta_{c}\right)\right)}{d \delta_{c}}\left(3 \delta_{h}-\bar{\delta}\right)+N P\left(\bar{G}\left(\delta_{h}\right)\right) g\left(\delta_{h}\right) .
\end{aligned}
$$

When $3 \delta_{h}-\bar{\delta} \geq 0, \frac{\partial^{2} S C}{\partial \delta_{h}^{2}} \geq 0$. So $\delta_{h}^{*}$ satisfies $\frac{\partial S C}{\partial \delta_{h}}=0$. 


\section{Proof of Lemma 2}

Similar to the proof of Proposition 1, it is easy to derive $q_{h}^{\prime *}=N\left[\bar{G}\left(\delta_{h}^{\prime *}\right)\right]$.

Then the problem becomes $\min S C\left(\left.\delta_{h}^{\prime}\right|_{q_{h}^{\prime *}}\right)=c N\left(\bar{G}\left(\delta_{h}^{\prime}\right)\right)+\int_{0}^{\delta_{h}^{\prime}} \delta N P\left(f^{\prime}\right) d G(\delta)$.

$\frac{\partial S C}{\partial \delta_{h}}=N g(\delta)\left(c-\delta_{h}^{\prime} P\left(\bar{G}\left(\delta_{h}^{\prime}\right)\right)\right)+N \frac{d P\left(\bar{G}\left(\delta_{h}^{\prime}\right)\right)}{d \delta_{h}^{\prime}} \int_{0}^{\delta_{h}^{\prime}} \delta d G(\delta)$. So $\delta_{h}^{\prime *}$ satisfies $c-$ $\delta_{h}^{\prime *} P\left(\bar{G}\left(\delta_{h}^{\prime *}\right)\right)<0$.

\section{Proof of Proposition 2}

$$
\frac{\partial \pi}{\partial q_{c}}= \begin{cases}r_{c}-c & q_{c} \leq N\left[\bar{G}\left(\delta_{c}\right)\right] \\ -c & q_{c}>N\left[\bar{G}\left(\delta_{c}\right)\right]\end{cases}
$$

So $q_{c}^{*}=N\left[\bar{G}\left(\delta_{c}\right)\right]$. Given $q_{c}^{*}, \pi\left(\left.\delta_{c}\right|_{q_{c}^{*}}\right)=\left(\delta_{c} P\left(\bar{G}\left(\delta_{c}\right)\right)-c\right) N\left[\bar{G}\left(\delta_{c}\right)\right]$.

$\frac{\partial \pi}{\partial \delta_{c}}=N g\left(\delta_{c}\right)\left(P\left(\bar{G}\left(\delta_{c}\right)\right)\left(\bar{\delta}-2 \delta_{c}\right)+\delta_{c}\left(\bar{\delta}-\delta_{c}\right) \frac{d P\left(\bar{G}\left(\delta_{c}\right)\right)}{d \delta_{c}}+c\right)$.

It is easy to derive $\frac{\partial \pi}{\partial \delta_{c}} \geq 0$ when $\bar{\delta}-2 \delta_{c} \geq 0$.

$\frac{\partial^{2} \pi}{\partial\left(\delta_{c}\right)^{2}}=N g\left(\delta_{c}\right)\left(-2 P\left(\bar{G}\left(\delta_{c}\right)\right)+2\left(\bar{\delta}-2 \delta_{c}\right) \frac{d P\left(\bar{G}\left(\delta_{c}\right)\right)}{d \delta_{c}}+\delta_{c}\left(\bar{\delta}-\delta_{c}\right) \frac{d^{2} P\left(\bar{G}\left(\delta_{c}\right)\right)}{d\left(\delta_{c}\right)^{2}}\right)$.

When $\bar{\delta}-2 \delta_{c}<0, \frac{\partial^{2} \pi}{\partial\left(\delta_{c}\right)^{2}} \leq 0$. So $\delta_{c}^{*}$ satisfies $\frac{\partial \pi}{\partial \delta_{c}}=0$.

\section{Proof of Lemma 3}

$$
\frac{\partial \pi}{\partial q_{s}}= \begin{cases}r_{s}+s-c & q_{s}<\left(N\left[\bar{G}\left(\delta_{s}\right)-\bar{G}(\beta)\right]\right)^{+} \\ -c & q_{s} \geq\left(N\left[\bar{G}\left(\delta_{s}\right)-\bar{G}(\beta)\right]\right)^{+}\end{cases}
$$

Then we find the optimal inventory

$$
q_{s}^{*}= \begin{cases}0 & \beta \leq \delta_{s}, \\ N\left[\bar{G}\left(\delta_{s}\right)-\bar{G}(\beta)\right] & \delta_{s}<\beta .\end{cases}
$$

The total profit is

$$
\pi\left(\delta_{s} \mid q_{s}^{*}\right)= \begin{cases}0 & \beta \leq \delta_{s}, \\ \left(\delta_{s} P\left(\bar{G}\left(\delta_{s}\right)\right)+s-c\right)\left(N\left[\bar{G}\left(\delta_{s}\right)-\bar{G}(\beta)\right]\right) & \delta_{s}<\beta .\end{cases}
$$

It is easy to derive $\left(\delta_{s} P\left(\bar{G}\left(\delta_{s}\right)\right)+s-c\right)\left(N\left[\bar{G}\left(\delta_{s}\right)-\bar{G}(\beta)\right]\right)>0$. So we have $\delta_{s}^{*}<\beta$. 


\section{Proof of Proposition 3}

$$
\begin{array}{r}
\frac{\partial \pi}{\partial \delta_{s}}=\frac{N}{\bar{\delta}}\left(P\left(\bar{G}\left(\delta_{s}\right)\right)\left(\beta-2 \delta_{s}\right)+\delta_{s}\left(\beta-\delta_{s}\right) \frac{d P\left(\bar{G}\left(\delta_{s}\right)\right)}{d \delta_{s}}+c-s\right), \\
\frac{\partial^{2} \pi}{\partial\left(\delta_{s}\right)^{2}}=\frac{N}{\bar{\delta}}\left(-2 P\left(\bar{G}\left(\delta_{s}\right)\right)+2\left(\beta-2 \delta_{s}\right) \frac{d P\left(\bar{G}\left(\delta_{s}\right)\right)}{d \delta_{s}}+\delta_{s}\left(\beta-\delta_{s}\right) \frac{d^{2} P\left(\bar{G}\left(\delta_{s}\right)\right)}{d\left(\delta_{s}\right)^{2}}\right) .
\end{array}
$$

When $c-s \geq 0, \frac{\partial \pi}{\partial \delta_{s}} \geq 0$ for $\delta_{s} \leq \frac{\beta}{2}$. When $\delta_{s} \geq \frac{\beta}{2}, \frac{\partial^{2} \pi}{\partial\left(\delta_{s}\right)^{2}} \leq 0$. So, for $c-s \geq 0$, $\delta_{s}^{*}$ satisfies $\frac{\partial \pi}{\partial \delta_{s}}=0$. Referring to $\frac{\partial^{2} \pi}{\partial\left(\delta_{s}\right)^{2}} \leq 0$ and $\frac{\partial \pi}{\partial \delta_{s}}$ increases with $\beta$, we can get that as $\beta$ increases, $\delta_{s}^{*}$ increases.

$s=P\left(\bar{G}\left(\delta_{s}\right)\right)\left(\beta-2 \delta_{s}\right)+\delta_{s}\left(\beta-\delta_{s}\right) \frac{d P\left(\bar{G}\left(\delta_{s}\right)\right)}{d \delta_{s}}+c$ for $s \leq c$, so $\frac{d s}{d \delta_{s}} \leq 0$.

When $c-s<0, \delta_{s}^{*}$ might be smaller than $\frac{\beta}{2}$.

\section{Proof of Proposition 4}

$\frac{d \int_{\delta_{c}}^{\beta} \delta d G(\delta)}{d \delta_{c}}=G\left(\delta_{c}\right)+\delta_{c} g\left(\delta_{c}\right)-G\left(\delta_{c}\right)=-\delta_{c} g\left(\delta_{c}\right) \leq 0 . \frac{d^{2} \int_{\delta_{c}}^{\beta} \delta d G(\delta)}{d\left(\delta_{c}\right)^{2}}=-g\left(\delta_{c}\right) \leq 0$.

By Proposition $3, h=\bar{G}\left(\delta_{s}^{*}\right)=\bar{G}(\beta)+\frac{q_{s}^{*}}{N}$ and

$s=P\left(\bar{G}\left(\delta_{s}^{*}\right)\right)\left(\beta-2 \delta_{s}^{*}\right)+\delta_{s}^{*}\left(\beta-\delta_{s}^{*}\right) \frac{d P\left(\bar{G}\left(\delta_{s}^{*}\right)\right)}{d \delta_{s}^{*}}+c$.

$\frac{d S C}{d s}=q_{s}^{*}+N \frac{d \delta_{s}^{*}}{d s}\left(\frac{d P(f)}{d \delta_{s}^{*}} \int_{0}^{\delta_{s}^{*}} \delta d G(\delta)+P(f) \delta_{s}^{*} g\left(\delta_{s}^{*}\right)\right)$.

$\frac{d^{2} S C}{d s^{2}}=N\left(\frac{d \delta_{s}^{*}}{d s}\right)^{2}\left(\frac{d^{2} P(f)}{d\left(\delta_{s}^{*}\right)^{2}} \int_{0}^{\delta_{s}^{*}} \delta d G(\delta)+2 \frac{d P(f)}{d \delta_{s}^{*}} \delta_{s}^{*} g\left(\delta_{s}^{*}\right)+P(f) g\left(\delta_{s}^{*}\right)\right) \geq 0$.

So $s^{*}$ satisfies $N\left[\bar{G}\left(\delta_{s}^{*}\right)-\bar{G}(\beta)\right]+N \frac{d \delta_{s}^{*}}{d s}\left(\frac{d P(f)}{d \delta_{s}^{*}} \int_{0}^{\delta_{s}^{*}} \delta d G(\delta)+P(f) \delta_{s}^{*} g\left(\delta_{s}^{*}\right)\right)=0$.

\section{Proof of Proposition 5}

$S C\left(q_{g}, \delta_{g}\right)=c\left(N[\bar{G}(\beta)]+q_{g}\right)+N P(f) \int_{0}^{\delta_{g}} \delta d G(\delta)+\left(1-\frac{q_{g}}{N\left[\bar{G}\left(\delta_{g}\right)-\bar{G}(\beta)\right]}\right)^{+} N P(f)$ $\int_{\delta_{g}}^{\beta} \delta d(G(\delta))$.

$$
\frac{\partial S C\left(q_{g}, \delta_{g}\right)}{\partial \delta_{g}}= \begin{cases}N \frac{d P\left(\bar{G}\left(\delta_{g}\right)\right)}{d \delta_{g}} \int_{0}^{\delta_{g}} \delta d G(\delta)+N P\left(\bar{G}\left(\delta_{g}\right)\right) \delta_{g} g\left(\delta_{g}\right) & \delta_{g} \geq \beta-\frac{Q_{g}}{N g(\delta)}, \\ \frac{N P(f) q_{g} g\left(\delta_{g}\right)}{N\left[\bar{G}\left(\delta_{g}\right)-\bar{G}(\beta)\right]}\left(\delta_{g}-\frac{1}{\bar{G}\left(\delta_{g}\right)-\bar{G}(\beta)} \int_{\delta_{g}}^{\beta} \delta d(G(\delta))\right) & \delta_{g}<\beta-\frac{Q_{g}}{N g(\delta)} .\end{cases}
$$

It is easy to derive $\frac{d P\left(\bar{G}\left(\delta_{g}\right)\right)}{d \delta_{g}} \int_{0}^{\delta_{g}} \delta d G(\delta)+P\left(\bar{G}\left(\delta_{g}\right)\right) \delta_{g} g\left(\delta_{g}\right) \geq 0$ and $\delta_{g}-\frac{1}{\bar{G}\left(\delta_{g}\right)-\bar{G}(\beta)}$ $\int_{\delta_{g}}^{\beta} \delta d(G(\delta))<0$. So $\delta_{g}^{*}=\beta-\frac{q_{g}}{N g(\delta)}$.

$h=\bar{G}(\beta)+\frac{q_{g}}{N}=\bar{G}\left(\delta_{g}^{*}\right)$, then we calculate $q_{g}^{*}, \frac{\partial S C\left(\left.q_{g}\right|_{\delta_{g}^{*}}\right)}{\partial q_{g}}=c+\left(\frac{d P(f)}{d f} \int_{0}^{\delta_{g}^{*}} \delta d G(\delta)\right.$ $\left.-P(f) \delta_{g}^{*}\right)$.

$\frac{\partial^{2} S C\left(\left.q_{g}\right|_{\delta_{g}^{*}}\right)}{\partial q_{g}^{2}}=\frac{1}{N}\left(\frac{d^{2} P(f)}{d f^{2}} \int_{0}^{\delta_{g}^{*}} \delta d G(\delta)-2 \delta_{g}^{*} \frac{d P(f)}{d f}+\frac{P(f)}{g\left(\delta_{g}\right)}\right) \geq 0$.

Then we find $q_{g}^{*}$ satisfying $c+\left(\frac{d P(f)}{d f} \int_{0}^{\delta_{g}^{*}} \delta d G(\delta)-P(f) \delta_{g}^{*}\right)=0$. 


\section{Proof of Corollary 4}

By Proposition 1, we have

$$
\begin{gathered}
-g\left(\delta_{h}^{*}\right)\left(P\left(\bar{G}\left(\delta_{h}^{*}\right)\right)\left(\bar{\delta}-2 \delta_{h}^{*}\right)+\delta_{h}^{*}\left(\bar{\delta}-\delta_{h}^{*}\right) \frac{d P\left(\bar{G}\left(\delta_{h}^{*}\right)\right)}{d \delta_{h}^{*}}+c\right) \\
+\left(\frac{d P\left(\bar{G}\left(\delta_{h}^{*}\right)\right)}{d \delta_{h}^{*}} \int_{0}^{\delta_{h}^{*}} \delta d G(\delta)+P\left(\bar{G}\left(\delta_{h}^{*}\right)\right) \delta_{h}^{*} g\left(\delta_{h}^{*}\right)\right)=0, \\
-g\left(\delta_{h}^{*}\right)\left(P\left(\bar{G}\left(\delta_{h}^{*}\right)\right)\left(\bar{\delta}-2 \delta_{h}^{*}\right)+\delta_{h}^{*}\left(\bar{\delta}-\delta_{h}^{*}\right) \frac{d P\left(\bar{G}\left(\delta_{h}^{*}\right)\right)}{d \delta_{h}^{*}}+c\right) \\
-g\left(\delta_{h}^{*}\right)\left(\frac{d P\left(\bar{G}\left(\delta_{h}^{*}\right)\right)}{d f} \int_{0}^{\delta_{h}^{*}} \delta d G(\delta)-P\left(\bar{G}\left(\delta_{h}^{*}\right)\right) \delta_{h}^{*}\right)=0,
\end{gathered}
$$

$P\left(\bar{G}\left(\delta_{h}^{*}\right)\right)\left(\bar{\delta}-2 \delta_{h}^{*}\right)+\delta_{h}^{*}\left(\bar{\delta}-\delta_{h}^{*}\right) \frac{d P\left(\bar{G}\left(\delta_{h}^{*}\right)\right)}{d \delta_{h}^{*}}+c+\frac{d P\left(\bar{G}\left(\delta_{h}^{*}\right)\right)}{d f} \int_{0}^{\delta_{h}^{*}} \delta d G(\delta)-P\left(\bar{G}\left(\delta_{h}^{*}\right)\right) \delta_{h}^{*}=0$.

By Proposition 5, we can easily derive Corollary 4.

\section{Proof of Corollary 5}

Under the vaccination subsidy scheme, we have

$$
\begin{aligned}
& N\left[\bar{G}\left(\delta_{s}^{*}\right)-\bar{G}(\beta)\right]+N \frac{d \delta_{s}^{*}}{d s}\left(\frac{d P\left(\bar{G}\left(\delta_{s}^{*}\right)\right)}{d \delta_{s}^{*}} \int_{0}^{\delta_{s}^{*}} \delta d G(\delta)+P\left(\bar{G}\left(\delta_{s}^{*}\right)\right) \delta_{s}^{*} g(\delta)\right)=0 . \\
& \frac{d P\left(\bar{G}\left(\delta_{s}^{*}\right)\right)}{d \delta_{s}^{*}} \int_{0}^{\delta_{s}^{*}} \delta d G(\delta)+P\left(\bar{G}\left(\delta_{s}^{*}\right)\right) \delta_{s}^{*} g(\delta)=-\frac{d s}{d \delta_{s}^{*}}\left(\bar{G}\left(\delta_{s}^{*}\right)-\bar{G}(\beta)\right) .
\end{aligned}
$$

Under the government allocation scheme, we have

$$
\begin{aligned}
& c+\frac{1}{N}\left(\frac{d P\left(\bar{G}\left(\delta_{g}^{*}\right)\right)}{d \bar{G}\left(\delta_{g}^{*}\right)} \int_{0}^{\delta_{g}^{*}} \delta d G(\delta)-P\left(\bar{G}\left(\delta_{g}^{*}\right)\right) \delta_{g}^{*}\right)=0 . \\
& c-\frac{1}{N g(\delta)}\left(\frac{d P\left(\bar{G}\left(\delta_{g}^{*}\right)\right)}{d \delta_{g}^{*}} \int_{0}^{\delta_{g}^{*}} \delta d G(\delta)+P\left(\bar{G}\left(\delta_{g}^{*}\right)\right) \delta_{g}^{*} g\left(\delta_{g}^{*}\right)\right)=0 . \\
& \frac{d P\left(\bar{G}\left(\delta_{g}^{*}\right)\right)}{d \delta_{g}^{*}} \int_{0}^{\delta_{g}^{*}} \delta d G(\delta)+P\left(\bar{G}\left(\delta_{g}^{*}\right)\right) \delta_{g}^{*} g(\delta)=c N g(\delta) .
\end{aligned}
$$

Setting $K(\hat{\delta})=\frac{d P(\bar{G}(\hat{\delta}))}{d \hat{\delta}} \int_{0}^{\hat{\delta}} \delta d G(\delta)+P(\bar{G}(\hat{\delta})) \hat{\delta} g(\delta)$, we have $\frac{d K(\hat{\delta})}{d \hat{\delta}} \geq 0$. Then it is easy to derive Corollary 5 .

\section{References}

Adida, E., Dey, D., \& Mamani, H. (2013). Operational issues and network effects in vaccine markets. European Journal of Operational Research, 231(2), 414-427.

Anderson, R. M., \& May, R. M. (1992). Infectious Diseases of Humans: Dynamics and Control. Oxford: Oxford University Press.

Arifoğlu, K., Deo, S., \& Iravani, S. M. (2012). Consumption externality and yield uncertainty in the influenza vaccine supply chain: Interventions in demand and supply sides. Management Science, 58(6), 1072-1091.

Arifoğlu, K., \& Tang, C. S. (2021). A two-sided incentive program for coordinating the influenza vaccine supply chain. Manufacturing \& Service Operations Management, available at: https://pubsonline.informs.org/ doi/10.1287/msom.2020.0938.

Aviv, Y., \& Pazgal, A. (2008). Optimal pricing of seasonal products in the presence of forward-looking consumers. Manufacturing \& Service Operations Management, 10(3), 339-359. 
Berenguer, G., Feng, Q., Shanthikumar, J. G., \& Xu, L. (2017). The effects of subsidies on increasing consumption through for-profit and not-for-profit newsvendors. Production and Operations Management, 26(6), 1191-1206.

Besley, T., \& Ghatak, M. (2001). Government versus private ownership of public goods. The Quarterly Journal of Economics, 116(4), 1343-1372.

Brito, D. L., Sheshinski, E., \& Intriligator, M. D. (1991). Externalities and compulsory vaccinations. Journal of Public Economics, 45(1), 69-90.

CDC. (2019). Influenza (flu). Retrieved October 20, 2020 from https://www.cdc.gov/flu/about/index.html.

Center of Health Protection (2020). Vaccination schemes: Persons aged 50 or above (seasonal influenza vaccine)/People aged 65 or above (pneumococcal vaccine). Retrieved Jan 292021 from https://www. chp.gov.hk/en/features/18881.html.

Cheng, L., Wong, N., \& Ng, K. (2020). Hong Kong private doctors already hit with flu vaccine shortages; 15 new Covid-19 cases confirmed. Retrieved Feb 72022 from https://www.scmp.com/news/hong-kong/ health-environment/article/3106085/demand-risesamid-covid-19-fears-hong-kongs.

Chick, S. E., Mamani, H., \& Simchi-Levi, D. (2008). Supply chain coordination and influenza vaccination. Operations Research, 56(6), 1493-1506.

Chief Secretary for Administration of the HKSAR (2021). Improve medical services without further ado. Retrieved Feb 72022 from https://www.cso.gov.hk/eng/blog/blog20210523.htm.

Cho, S.-H., \& Tang, C. S. (2013). Advance selling in a supply chain under uncertain supply and demand. Manufacturing \& Service Operations Management, 15(2), 305-319.

Dai, T. (2015). Incentives in USA healthcare operations. Decision Sciences, 46(2), 455-463.

Dai, T., Cho, S.-H., \& Zhang, F. (2016). Contracting for on-time delivery in the us influenza vaccine supply chain. Manufacturing \& Service Operations Management, 18(3), 332-346.

Demirci, E. Z., \& Erkip, N. K. (2020). Designing intervention scheme for vaccine market: A bilevel programming approach. Flexible Services and Manufacturing Journal, 32(2), 453-485.

Deng, H., Wang, Q., Leong, G. K., \& Sun, S. X. (2008). The usage of opportunity cost to maximize performance in revenue management. Decision Sciences, 39(4), 737-758.

Deo, S., \& Corbett, C. J. (2009). Cournot competition under yield uncertainty: The case of the USA influenza vaccine market. Manufacturing \& Service Operations Management, 11(4), 563-576.

Duijzer, L. E., van Jaarsveld, W., \& Dekker, R. (2018). Literature review: The vaccine supply chain. European Journal of Operational Research, 268(1), 174-192.

Erhun, F., Keskinocak, P., \& Tayur, S. (2008). Dynamic procurement, quantity discounts, and supply chain efficiency. Production and Operations Management, 17(5), 543-550.

Galvani, A. P., Reluga, T. C., \& Chapman, G. B. (2007). Long-standing influenza vaccination policy is in accord with individual self-interest but not with the utilitarian optimum. Proceedings of the National Academy of Sciences, 104(13), 5692-5697.

Hong Kong Government News (2020a). Government allocates seasonal influenza vaccines to facilitate vaccination for high risk groups. Retrieved Feb 72021 from https://www.info.gov.hk/gia/general/202010/ 22/P2020102200888.htm?fontSize=1.

Hong Kong Government News (2020b). Vaccine Subsidy Scheme 2020/21 to start this Thursday. Retrieved Jan 292021 from https://www.info.gov.hk/gia/general/202010/05/P2020100500384.htm?fontSize=1.

Iossa, E., \& Martimort, D. (2015). The simple microeconomics of public-private partnerships. Journal of Public Economic Theory, 17(1), 4-48.

Kivleniece, I., \& Quelin, B. V. (2012). Creating and capturing value in public-private ties: A private actor's perspective. Academy of Management Review, 37(2), 272-299.

Lin, Q., Zhao, Q., \& Lev, B. (2022). Influenza vaccine supply chain coordination under uncertain supply and demand. European Journal of Operational Research, 297(3), 930-948.

Mamani, H., Adida, E., \& Dey, D. (2012). Vaccine market coordination using subsidy. IIE Transactions on Healthcare Systems Engineering, 2(1), 78-96.

Meltzer, M. I., Cox, N. J., \& Fukuda, K. (1999). The economic impact of pandemic influenza in the united states: Priorities for intervention. Emerging Infectious Diseases, 5(5), 659.

Murray, J. D. (1993). Mathematical Biology. Berlin: Springer.

Pan, Y., Ng, C. T., \& Cheng, T. C. E. (2021). Effect of free-riding behavior on vaccination coverage with customer regret. Computers \& Industrial Engineering, 159, 107494.

Su, X., \& Zhang, F. (2008). Strategic customer behavior, commitment, and supply chain performance. Management Science, 54(10), 1759-1773.

Tuck, L., \& Lindert, K. (1996). From Universal Food Subsidies to a Self-Targeted Program: A Case Study in Tunisian Reform. The World Bank.

Wikipedia (2018). Basic reproduction number. https://en.wikipedia.org/wiki/Basic_reproduction_number. 
Xie, L., Hou, P., \& Han, H. (2021). Implications of government subsidy on the vaccine product R\&D when the buyer is risk averse. Transportation Research Part E: Logistics and Transportation Review, 146, 102220.

Publisher's Note Springer Nature remains neutral with regard to jurisdictional claims in published maps and institutional affiliations. 